\title{
Electrolyte Levels Analysis on Diabetes Mellitus Patients in Noongan Regional General Hospital, North Sulawesi, Indonesia
}

\author{
Diana Shintawati Purwanto ${ }^{1,2,}$, , Yanti Meilen Mewo ${ }^{1}$, Edmond Leonard $\mathrm{Jim}^{3}$, \\ Richardo Jordan Laloan ${ }^{4}$, Hessyani Patrisia Theodora Raranta ${ }^{2}$, Billy Johnson Kepel ${ }^{5}$ \\ ${ }^{1}$ Department of Biochemistry, Faculty of Medicine, Sam Ratulangi University, Manado, Indonesia \\ ${ }^{2}$ Department of Clinical Laboratory, R. D Kandou Central General Hospital, Manado, Indonesia \\ ${ }^{3}$ Department of Cardiovascular Medicine, Faculty of Medicine, Sam Ratulangi University, Manado, Indonesia \\ ${ }^{4}$ Clinical Clerkship Program, Faculty of Medicine, Sam Ratulangi University, Manado, Indonesia \\ ${ }^{5}$ Department of Chemistry, Faculty of Medicine, Sam Ratulangi University, Manado, Indonesia
}

Email address:

dianashintapurwanto@unsrat.ac.id (D. S. Purwanto)

${ }^{*}$ Corresponding author

\section{To cite this article:}

Diana Shintawati Purwanto, Yanti Meilen Mewo, Edmond Leonard Jim, Richardo Jordan Laloan, Hessyani Patrisia Theodora Raranta, Billy Johnson Kepel. Electrolyte Levels Analysis on Diabetes Mellitus Patients in Noongan Regional General Hospital, North Sulawesi, Indonesia. International Journal of Diabetes and Endocrinology. Vol. 5, No. 4, 2020, pp. 54-60. doi: 10.11648/j.ijde.20200504.11

Received: October 16, 2020; Accepted: November 16, 2020; Published: November 19, 2020

\begin{abstract}
Diabetes mellitus is a metabolic disease characterized by hyperglycemia caused by defects in insulin secretion, insulin action, or both, that affect the action of insulin on the target tissue. North Sulawesi is in the top 5 of 34 provinces in Indonesia with the highest prevalence of diabetes mellitus, based on the results of the 2018 Basic Health Research. The relationship between blood glucose and electrolytes is very complex, so there are still few studies looking for the relationship between the two in diabetes mellitus patients. This study aimed to find out the association between electrolytes levels (serum sodium, potassium, chloride, and magnesium) and fasting blood glucose in type 2 diabetes mellitus patients. This cross-sectional study was conducted in April-September 2020 at Noongan Regional Hospital, North Sulawesi, Indonesia. Demographic data including age, sex, symptoms, and oral anti-diabetic medication/insulin administration were collected, and then physical examination including body mass index was carried out. The Kolmogorov-Smirnov and Spearman Rank tests were used for statistical analysis. There is an inverse correlation between fasting blood glucose and serum sodium, chloride, and magnesium, but not significant. As for potassium, there is a direct correlation, but also not significant. In conclusion, only potassium has a positive correlation with fasting blood glucose, while other electrolytes have a negative correlation, however all of correlations are not significant.
\end{abstract}

Keywords: Diabetes Mellitus, Electrolyte, Blood Glucose

\section{Introduction}

Diabetes mellitus (DM) is a progressive disease that has a multifactorial etiology and is characterized by chronic hyperglycemia and metabolic disorders of carbohydrates, fat, and proteins as a result of relative or absolute defects of islet $\beta$-cell pancreas, causing impaired insulin secretion or action, or both $[1,2]$ Nowadays, diabetes mellitus is becoming one of the common non-communicable diseases worldwide. It is estimated that deaths due to diabetes mellitus have occurred as many as 1.5 million deaths globally. Approximately, half of the deaths in the data are due to chronic hyperglycemia occurring before the age of 70 . WHO predicts diabetes mellitus will be the $7^{\text {th }}$ disease that causes death in 2030 . According to the International Diabetes Federation in 2017, the incidences of diabetes mellitus is estimated to affect 451 million people globally and is projected to increase to 693 million people with diabetes by $2045[3,4]$. 
In Indonesia, there are nearly 133 million people suffering from diabetes mellitus, and about $87.5 \%$ of patients do not meet the goal target of glycemic control properly [5]. According to the established data that has been determined from the 2018 Indonesian Basic Health Research, the prevalence of diabetes mellitus in Indonesia in 2018 based on age shows that age 55-64 years is the highest, followed by the age group of 65-74 as the second highest case. Meanwhile, the proportion based on gender shows that women are more likely to suffer from diabetes mellitus than men. This prevalence affects people who live in urban more than in rural areas. The prevalence of diabetes mellitus based on physician's diagnosis among Indonesian residents in all age group by province shows that North Sulawesi is in top 5 of a total of 34 provinces [6].

Diabetes mellitus and its complications significantly increase financial burden on the family and public health, which implies a significant increase in the burden of country health costs and also an increase in disability which leads to a decrease in life expectancy and low quality of life [5]. Chronic hyperglycemia can cause complications to the eyes, kidneys, and nerves, but also increases the risk of cardiovascular disease. Complication of diabetes mellitus can lead to vascular degeneration, metabolic imbalance, and can cause alterations of electrolytes concentrations in the human body. Electrolyte have an important role in maintaining acid-base balance, body fluid homeostasis, blood clotting, and muscle contraction. Diabetic nephropathy is one of the complications that can cause renal failure and this condition can lead to electrolyte imbalance in diabetic patients. A disrupted electrolyte distribution process can affect the course of the disease and also its management at that time. Electrolytes and blood glucose have complex relationships and are linked to a number of factors, such as age and underlying conditions. Several factors as pathomechanisms such as nutritional status, concomitant acid-base disturbances, the effect of some drugs and other comorbidities such as renal impairement or the sum of those factors aforementioned can also play an important role in electrolyte imbalance [7].

Three specific abnormalities in diabetes mellitus are insulin secretion disturbance, increased hepatic glucose production, and decreased insulin-stimulated glucose uptake in peripheral tissues. Glucose is an active osmotic substance, and in some condition of hyperglycemia can increase serum osmolality which can lead to water shift to outer cell; and there is a decrease in sodium levels because of dilution effect. The osmotic effect of glucose can also cause osmotic diuresis resulting in a decrease in circulating blood volume, as well as cellular dehydration due to water shift from the intracellular space [8].

Diabetes mellitus is a disease with independent risk factors that can cause either hyponatremia or hypernatremia through several underlying mechanisms. Uncontrolled diabetes mellitus can induces hypovolemic hyponatremia due to osmotic diuresis phenomenon. In diabetic ketoacidosis, the condition of electrolyte loss through urine can increase sodium wasting from the kidneys. Increased chloride serum levels can also be seen in diabetic patients. This condition can be caused by diabetic ketoacidosis which causes a decrease in blood $\mathrm{pH}$ and further disrupts the acid-base balance and leads to an increase in chloride levels [9].

The relationship between blood glucose and electrolytes is very complex, so there are still few studies looking for the relationship between the two in diabetes mellitus patients. Therefore, this study aimed to determine and analyze the correlation between electrolytes levels and high blood fasting glucose in patients diagnosed with diabetes mellitus in Noongan Regional General Hospital, Minahasa Regency.

\section{Material and Methods}

\subsection{Ethical Approval}

This study was approved by the Medical Research Ethics Committee of R. D. Kandou General Hospital (Ethical Approval number 071/EC/KEPK-KANDOU/VIII/2020). Written informed consent for participating in the study was obtained from all of the patients or accompanying close relatives.

\subsection{Study Design and Study Timeline}

A cross-sectional study was conducted at Noongan Regional General Hospital, Minahasa Regency. This study site is a regency hospital located around $50 \mathrm{~km}$ from Manado, the capital city of North Sulawesi, at the northeastern part of Indonesia. This study only analyzed the correlation between fasting blood glucose of diabetes mellitus patients and electrolyte levels, without giving any interventions or treatments to all subjects. The timeline of sample collection was started in April 2020 and finished in September 2020.

\subsection{Populations and Sampling Methodology}

Populations were all inpatients and outpatients at the Noongan Regional General Hospital, Minahasa Regency with a diagnosis of diabetes mellitus and had high fasting blood glucose levels ( $>126 \mathrm{mg} / \mathrm{dL})$. Sample collection used total sampling method, in which included all the subjects who were examined during the study period and met the inclusion and exclusion criteria and willing to participate in this study. A prior written approval was given by participant before undergoing the procedure.

\subsection{Statistical Data Analysis}

Data analysis was performed by using 22nd version of Statistical Package for Social Sciences (SPSS 22). Univariate analysis was conducted to have data results descriptively of dependent variable (fasting blood glucose) and independent variables (sodium, potassium, chloride and magnesium). Meanwhile, bivariate analysis was conducted to determine the correlation between dependent and independent variables. The normality of each variable was calculated using the Kolmogorov-Smirnov One Sample test, and it had a normal distribution if the value of $p>0.05$. Subsequently, the correlation between dependent and independent variables was examined using Spearman's rank analysis test. 


\section{Results}

\subsection{Demographic Data}

The patient characteristics are described in Table 1 . Seventy-two blood specimens were collected from patients with clinically diagnosed as diabetes mellitus. The age of the patients is between 38 to 77 years and the majority is $31.94 \%$ $(n=23)$ with aged 55-64 years, with an average age of $58.2 \pm 10.17$ years. Thirty-four of 72 patients are male (47.2\%). It was found that the majority of patient symptoms were fever (36.1\%), headache (31.9\%), and dizziness (20.8\%). We observed that majority of subjects had normal Body Max Index $(73.6 \%)$.

Table 1. Patient characteristics (demographic and clinical).

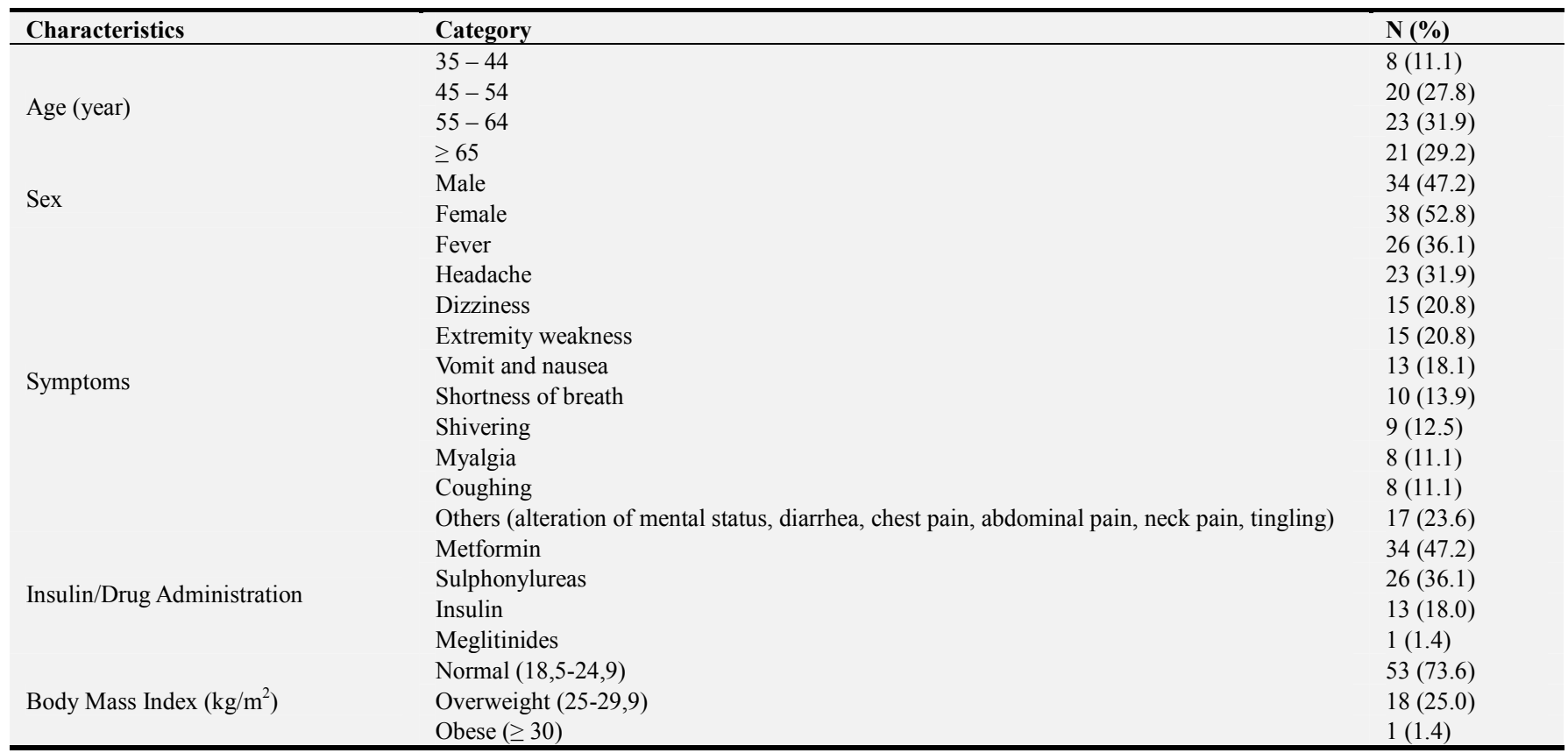

\subsection{Glucose, Sodium, Potassium, Chloride and Magnesium Levels}

Table 2 shows the mean and median of glucose, sodium, potassium, chloride, and magnesium electrolyte concentrations. Based on statistical data, the potassium concentration had a normal distribution, while glucose, sodium, chloride, and magnesium had an abnormal distribution data.

Table 2. Glucose, sodium, potassium, chloride, and magnesium levels.

\begin{tabular}{|c|c|c|c|c|c|c|}
\hline Electrolytes & Unit & Mean & Median & Standard deviation & Minimum & Maximum \\
\hline Glucose & $\mathrm{mg} / \mathrm{dL}$ & 246.2 & 214 & 92.1 & 148 & 500 \\
\hline Sodium & $\mathrm{mg} / \mathrm{dL}$ & 136.6 & 137.5 & 8.6 & 109 & 161 \\
\hline Potassium & $\mathrm{mg} / \mathrm{dL}$ & 4.2 & 4.1 & 0.8 & 2.0 & 5.9 \\
\hline Chloride & $\mathrm{mg} / \mathrm{dL}$ & 101.4 & 102 & 8.9 & 76 & 123 \\
\hline Magnesium & $\mathrm{mg} / \mathrm{dL}$ & 1.88 & 1.83 & 0.64 & 0.53 & 5.15 \\
\hline
\end{tabular}

\subsection{Correlation Test Between Fasting Blood Glucose and Electrolyte Levels}

The correlation test between the glucose and electrolytes (sodium, potassium, chloride, and magnesium) levels in diabetes mellitus patients used the Spearman's rank correlation test. The results of this correlation are described in Table 3.

Table 3. Correlation test results between fasting blood glucose and electrolytes levels.

\begin{tabular}{lll}
\hline \multirow{2}{*}{ Electrolytes } & Glucose & Sig. $(\mathbf{p})$ \\
\cline { 2 - 3 } & Correlation coefficient (rs) & 0.606 \\
\hline Sodium & -0.062 & 0.526 \\
Potassium & 0.076 & 0.487 \\
Chloride & -0.083 & 0.203 \\
Magnesium & -0.152 & \\
\hline
\end{tabular}

It is shown in Table 3 that the correlation between glucose and sodium levels has a value of $\mathrm{rs}=-0.062$ which can be categorized as having a very weak and negative correlation (inversely proportional), which means that if glucose levels in 
blood serum are high then sodium levels will decrease. Based on the significance test, the result shows $p$-value $=0.606$ ( $p>0.05$ ) then the null hypothesis is accepted, meaning that there is no significant relationship between glucose and sodium concentrations.

The correlation between glucose and potassium levels has a value of $r s=0.076$ which can be categorized as having a very weak and positive correlation (directly proportional), which means that if the glucose levels in blood serum are high then the potassium levels will increase. Based on the significance test, the result shows $p$-value $=0.526(\mathrm{p}>0.05)$ and so the null hypothesis is accepted, meaning that there is no significant relationship between glucose and potassium concentrations.

The correlation of glucose and chloride levels has a value of $\mathrm{rs}=-0.083$ which can be categorized as having a very weak and negative correlation (inversely proportional), which means that if glucose levels in blood serum are high then chloride levels will decrease. Based on the significance test, the result shows $p$-value $=0.487(\mathrm{p}>0.05)$ and so the null hypothesis is accepted, meaning that there is no significant relationship between glucose and chloride concentrations.

The correlation of glucose and magnesium levels has a value of $r s=-0.152$ which can be categorized as having a weak and negative correlation strength (inversely proportional), which means that if the glucose levels in blood serum are high then the magnesium levels will decrease. Based on the significance test, the result shows $p$-value $=0.203$ ( $\mathrm{p}>0.05)$, and so the null hypothesis is accepted, meaning that there is no significant relationship between glucose and magnesium concentrations.

\section{Discussion}

The result of this study descriptively shows that the prevalence of diabetes mellitus is more likely suffered mostly by subject age 55-64 years-old and the least are those in the age range of 35-44 years-old. Similar to our findings, there is a sociodemographic characteristic analysis shows that people with diabetes mellitus increased a risk with age where the highest proportion has been found out in people age more than or equal to 55 years old [10].

Older people with diabetes are associated with increased risk of frailty which characterized by multisystem dysregulation leading to a decrease in physiological function and a further increase in health risk. This dysregulation also affects innate and adaptive immunity as well as increases in inflammatory markers and susceptibility to infection. Frailty is generally associated with infectious diseases through several pathways that reduce immunity [11]. This path will be discussed further in this section.

The sociodemographic analysis also reported that there is no significant difference between the proportion of sex, male and female in terms of the incidence of DM [10]. This is contradictive with our findings in which our results indicate that the prevalence of diabetes mellitus is more likely in females than males. Our study also revealed that most of the subjects had normal BMI. This is in line with an analytical study by Sobers-Grannum, N. et al conducted in the Caribbean region which confirmed that females are more likely to suffer from diabetes mellitus than males, but however these findings may differ in other parts of the world [12].

In general, males are more likely to suffer from type 2 diabetes mellitus at a younger age than females. In addition, there are predictor factors that increase the susceptibility of both sexes to type 2 diabetes mellitus. In females with a higher $\mathrm{BMI}$, it is a predictor of an increased tendency to suffer from diabetes mellitus, while in males the predictor is waist circumference In males, the frequently finding is abdominal obesity, whereas females can be in a state of so-called metabolically healthy obesity. In the pre-diabetic stage, a fasting hyperglycemia state is frequently seen in males, whereas a glucose intolerance state is seen more frequently in females [13].

Furthermore, based on clinical characteristics, it was found that $60 \%$ of diabetes mellitus patients in Indonesia have at least one co-morbid underlying diabetes mellitus [10]. Inadequate physical activity refers to the development of type 2 diabetes mellitus in women, whereas the condition of the metabolic syndrome with hypertension and dyslipidemia is more common in men. Smoking is a risk factor for both sexes, however in the case of women who smoke and have type 2 diabetes mellitus have significantly higher cardiovascular complications than men who smoke and have type 2 diabetes mellitus $[12,13]$.

In this recent study, we found out that the most symptom of diabetes mellitus patients is fever. In theory, diabetes mellitus is associated with a decreased response of T-cells formation, neutrophil function, and impaired humoral immunity. As a result, people with diabetes mellitus are increasingly susceptible to infection. The pathogenesis of infectious susceptibility can be explained in a number of mechanisms. First, the compromised complement system, where we know that this system is one of the main mechanisms responsible for the activation of humoral immunity. This system involves serum and surface proteins which have the main function to promote opsonization and phagocytosis of microorganisms via macrophages and neutrophils and induce the lysis of microorganisms [14]. Furthermore, this complement system activates products and provides secondary signals to activate B-lymphocytes and produce antibodies. In several studies, there has been a deficiency of the $\mathrm{C} 4$ component in people with mellitus, where $\mathrm{C} 4$ depletion is thought to be associated with polymorphonuclear dysfunction (PMN) and decreased cytokine response [15].

Second, it turns out that patients with diabetes mellitus, mononuclear cells and monocytes secrete less interleukin-1 (IL-1) and interleukin-6 (IL-6) in response to stimulation by lipopolysaccharides. Low interleukin production is thought to be due to intrinsic cell defects in people with diabetes. Furthermore, several studies have shown that increased glycation can inhibit the production of IL-10 from myeloid cells, as well as interferon gamma (IFN- $\gamma$ ) and tumor necrosis factor alpha (TNF- $\alpha$ ) from T-cells. Glycation can also decrease the expression of the major histocompatibility 
complex ( MHC) class I on the surface of myeloid cells resulting in damage to immune cells $[14,15]$.

Third, there is a suppressing immune response which causes a decrease in the mobility of PMN leukocytes, chemotaxis and phagocytic activity that can occur in hyperglycemia conditions. This hyperglycemic condition can also block antimicrobial function by inhibiting glucose-6-phosphate dehydrogenase (G6PD) and increasing PMN leukocyte apoptosis and decreasing its transmigration to the endothelium. In tissues that do not need insulin to transport glucose, this hyperglycemia condition increases intracellular glucose levels and is further metabolized using NADPH as a cofactor in this process. This decrease in NADPH prevents the regeneration of a molecule that plays a key role in the antioxidant mechanism in cells. This process increases the susceptibility to oxidative stress $[14,15]$.

In a previous study by Rajagambeeram, R. et al. reported that there was an inverse correlation between serum sodium levels in diabetic patients. The results showed that sodium levels had a negative correlation with fasting blood glucose levels and HbA1C levels. On the other hand, potassium levels have a positive correlation with fasting blood glucose, but not significant. Hyperkalemia has clearly shown an association with hyperglycemia [16]. The results of the inverse correlation also support our findings in this recent study. It was found that sodium and potassium levels were not significantly correlated with blood glucose, but for potassium levels, it was positively correlated with hyperglycemia, in which it is proper with the existing theory

Another study by Karuppa, A. et al showed that a common electrolyte abnormality in DM is hyponatremia, in which about one third of subjects reported had low sodium levels $(<130 \mathrm{mEq} / \mathrm{L})$. This supports the existing theory that patients with poor blood glucose control have a higher risk for hyponatremia due to the inability of the kidneys to maintain control homeostatic mechanisms which include thirst, secretion of antidiuretic hormone (ADH), and renal function to control filtering of sodium excretion [17].

The frequency of hyponatremia is more likely to be seen in the elderly than in younger ages. Drugs may also cause hyponatremia in diabetic patients. A number of drugs are associated with a high risk of hyponatremia, including diuretics, selective serotonin reuptake inhibitors (SSRIs), angiotensin-converting-enzyme inhibitors (ACE-i), opioids and anti-arrhythmic drugs. However, drug-induced hyponatremia is more common as a result of chronic drug use. In addition, each hospitalized patient has the possibility of electrolyte correction as part of hospital management to prevent potential risk of mortality in diabetic patients [17].

In uncontrolled diabetic patients, there is a phenomenon called pseudohyponatremia which is having low sodium concentrations, normal serum osmolality, but experiencing hypertriglyceridemia. This condition requires proper diagnosis to prevent the dangers of overtreatment. Therefore, in such cases, the patient does not show classic symptoms in relation to hyponatremia. This condition does not require treatment to correct sodium but requires adequate glycemic control, as this glycemic control is associated with decreased serum triglycerides and results in correction of pseudohyponatremia [18].

The main cause of hypotonic hyponatremia in diabetic patients is due to osmottic diuresis-induced hypovolemia. In diabetic ketoacidosis (DKA) patients, obligate urinary $\beta$-hydroxybutyrate and acetoacetate sodium acetoacetate are excreted which results in decreased hypovolemia. The alteration of vasopressin hormone in DM with renal complication leads to an increase in the expression of insulin-induced aquaporin (AQP-2) water channels and water absorption in the gastrointestinal tract (GI) due to slower gastric emptying. These are considered to play a key role in the association between diabetes mellitus and hyponatremia $[17,18]$.

Study by Rajagambeeram, R. et al also reported that diabetic patients were more likely to develop hypokalemia than hyperkalemia, but there were no significant correlations and differences between uncontrolled and controlled subjects [17]. Hypokalemia in diabetics can be caused by several mechanisms. First, there is a shift in the redistribution of potassium from the extracellular fluid (ECF) compartment to intracellular fluid (ICF) due to the use of insulin. Second, the loss of potassium from the GI tract due to malabsorption syndrome or impaired motility due to diabetes which causes bacterial overgrowth and causes chronic diarrhea. Third, loss of potassium from the renal system due to osmotic diuresis, or preexisting hypomagnesemia. The fact that hypomagnesemia causes hypokalemia due to low intracellular concentrations of magnesium $(\mathrm{Mg} 2+)$ leads to activation of extracellular medullary $\mathrm{K}+$ channels to be excreted more [18].

This recent study revealed that chloride (Cl-) was negatively correlated with glucose levels in diabetic patients and had a very weak correlation. In a study conducted by Woyesa, SB, et al. demonstrated a correlation between chloride and as risk factors for diabetes mellitus. Three electrolyte abnormalities were found in diabetics, namely hyponatremia, hypochloremia and hyperkalemia. Several previous studies have also identified a condition in diabetic patients, called diabetic hyperchloremia. These serum electrolyte abnormalities were found to accompany sodium and potassium abnormalities [19].

Furthermore, Woyesa, SB, et al found that the correlation between abnormal serum chloride concentration levels and drug type for diabetic patients was negative and very weak for the correlation coefficient [19]. This contradicts Kataoka, H, et al. which investigated the effects and possible mechanisms underlying the diuretics of the sodium-glucose cotransporter 2 inhibitor (SGLT2i) drug on serum chloride concentrations and were clinically significant based on the "chloride theory" in type $2 \mathrm{DM}$ and type 2 heart failure (HF) patients. The study postulated that, according to the "chloride theory", chloride ion is the main key of electrolytes to regulate and distribute plasma volume in all body spaces, namely the intracellular, intravascular, interstitial compartments and pleural spaces; and not sodium. Therefore, compared to cationic sodium ions, anionic chloride ions in the human body have the potential for 
"tonicity" in the vascular space, thus fueling the concept of this theory [20].

The study also showed that medical treatment for diabetes mellitus using SGLT2i drugs can be classified as a "chloride-regaining diuretics" to preserve or enhance chloride serum concentration via a number of mechanisms. According to the concept of "chloride-theory" and SGLT2i as "chloride-regaining", it can be considered that this combination could have peculiar properties to preserve plasma volume and renal function, also the capability draining interstitial body fluid by serum chloride-associated enhancement of vascular tonicity. This concept is consistent with clinical observation nowadays that SGLT2i reduces interstitial congestion without any deleterious effects of arterial underfilling or predominantly decreases extracellular volume. However, it is important to remember that SGLT2i cannot be administered to diabetic patients and non-HF patients with hypernatremia and hyperchloremia [20].

Magnesium $(\mathrm{Mg} 2+)$ is involved in the process of insulin secretion and acts as a cofactor in various enzyme processes for carbohydrate metabolism. Magnesium deficiency decreases the affinity of glucose for binding to glucokinase and indirectly results in impaired insulin secretion, insulin resistance and leads to increased macrovascular risk. Numerous studies have documented that hypomagnesemia is associated with poor glycemic control, and possibly due to urinary wastage of magnesium. This study revealed that magnesium was negatively correlated with glucose levels in diabetic patients and had a weak correlation. Research conducted by Rajagambeeram, $\mathrm{R}$, et al. found that magnesium levels were negatively correlated with glycemic control and fasting blood glucose (FBG), and the strength of the correlation was found to be not strongly correlated. Hypomagnesemia is an electrolyte disorder due to nutritional deficiencies, diuretics, metabolic acidosis, glomerular hyperfiltration, and changes in insulin metabolism [16].

Epidemiological studies suggest that low magnesium intake is associated with an increased risk of developing diabetes mellitus, whereas high magnesium intake is associated with a reduced risk of diabetes mellitus. In addition, hypomagnesemia also causes impaired glucose disposal and contributes to cardiovascular disease, retinopathy and nephropathy. The incidence of hypomagnesemia in people with type 2 diabetes mellitus is very wide, and depends on the cause, such as oral pool intake and chronic diarrhea associated with autonomic neuropathy. Proton pump inhibitor drugs (PPIs) also interfere with magnesium absorption in the GI tract. This effect results from a drug-induced low $\mathrm{pH}$ in the intestinal lumen which alters the affinity of potential transient receptors namely melastatin-6 and melastatin-7 (TRPM6 and TRPM7) channels on the apical surface of enterocytes for magnesium [21].

This study has several limitations, including the size of the research subjects is not large enough for the results of electrolytes to be statistically significant. This could be due to the fact that the period of sample collection was during the Covid-19 pandemic so that the number of patients visiting the hospital was significantly reduced.

\section{Conclusion}

The risk of developing diabetes mellitus increases with age, especially in those who are over or equal to 55 years of age, are female, and have an excess body mass index. Only potassium has a positive correlation with fasting blood glucose, whereas other electrolytes have negative correlation, however all correlations are not significant. Because four electrolyte abnormalities are found in people with diabetes mellitus, namely hyponatremia, hyperkalemia, hypochloremia, and hypomagnesemia, therefore electrolyte examination should be routinely measured in type 2 diabetes.

\section{Conflict of Interest Statement}

All the authors do not have any possible conflicts of interest

\section{Acknowledgements}

This study was fully funded by Directorate Research and Community Service, Sam Ratulangi University, Manado, Indonesia. The authors would like to thank the Noongan Regional General Hospital for all the support and also to all patients who participated in this study.

\section{References}

[1] Alberti, KG., Zimmet, PZ. Definition, Diagnosis and Classification of Diabetes Mellitus and Its Complications. Part 1: Diagnosis and Classification of Diabetes Mellitus Provisional Report of A WHO Consultation. Diabet Med. 1998; Vol. 15 (7): p. 539-553.

[2] American Diabetes Association. Classification and Diagnosis of Diabetes: Standards of Medical Care in Diabetes -2020 . Diabetes Care. 2020; Vol. 43 (1): p. S14-S31.

[3] Khan, RN., Saba, F., Kausar, SF., Siddiqui, MH. Pattern of Electrolyte Imbalance in Type 2 Diabetes Patients: Experience from A Tertiary Care Hospital. Pak J Med Sci. 2019; Vol. 35 (3): p. $797-801$.

[4] Wei, D., Liu, X., Jiang, J., Tu, R., Qiao, D., Li, R., et al. Mineralocorticoids, Glucose Homeostasis and Type 2 Diabetes Mellitus: The Henan Rural Cohort study. J Diabetes Complications. 2020: Vol. 34 (5): p. 107558.

[5] Pamungkas, RA., Chamroonsawasdi, K. Self-Management Based Coaching Program to Improve Diabetes Mellitus Self-Management Practice and Metabolic Markers Among Uncontrolled Type 2 Diabetes Mellitus in Indonesia: A Quasiexperimental Study. Diabetes Metab Syndr. 2020; Vol. 14: p. 53-61.

[6] Riset Kesehatan Dasar (RISKESDAS). Prevalensi Diabetes Melitus Berdasarkan Diagnosis Dokter pada Penduduk Semua Umur Menurut Provinsi. Kementerian Kesehatan RI Badan Penelitian dan Pengembangan Kesehatan. 2018; p. 67-68. 
[7] Valarmathi, A., Sastri, L. Evaluation of Electrolytes Level Imbalance as a Risk Factor for Chronic Complications in Diabetic Patients. Sch J App Med Sci. 2017; Vol. 5 (7E): p. 2934-2936.

[8] Pragna, D. A Study of Serum Electrolyte Levels and Lipid Profile in Chronic Type 2 Diabetes Mellitus. Saudi J. Med. 2018; Vol. 3 (8): p. 477-481.

[9] Datchinamoorthi, S., Vanaja, R., Rajagopalan, B. Evaluation of Serum Electrolytes in Type II Diabetes Mellitus. Int J Pharm Sci Rev Res. 2016; Vol. 40 (1): p. 251-253.

[10] Arifin, B., Perwitasari, DA., Thobari, JA., Cao, Q., Krabbe, PFM., Postma, MJ. Translation, Revision, and Validation of the Diabetes Distress Scale for Indonesian Type 2 Diabetic Outpatients with Various Types of Complications. Value Health Reg Issues. 2017; Vol. 12C: p. 63-73.

[11] Sinclair, AJ., Abdelhafiz, AH. Age, Frailty and Diabetes Triple Jeopardy fot Vulnerability to COVID-19 Infection: Commentary. EClinicalMedicine. 2020; Vol. 22: p. 100334.

[12] Sobers-Grannum, N., Murphy, MM., Nielsen, A., Guell, C., Samuels, TA., Bishop, L., et al. Female Gender Is a Social Determinant of Diabetes in the Caribbean: A Systematic Review and Meta-Analysis. PLoS ONE. 2015; Vol. 10 (5): p. e0126799.

[13] Dedinská, I., Graňák, K., Vnučák, M., Skálová, P., Kováčiková, L., Laca, L., et al. Role of Sex In Post-Transplant Diabetes Mellitus Development: Are Men and Women Equal? J Diabetes Complications. 2019; Vol. 33 (4): p. 315-322.

[14] Casqueiro, J., Casqueiro, J., Alves, C. Infection in Patients with Diabetes Mellitus: A Review of Pathogenesis. Indian J Endocrinol Metab. 2012; Vol. 16 (1): p. S27-S36.
[15] Erener, S. Diabetes, Infectiom Risk and COVID-19: A Review. Mol Metab. 2020; Vol. 39: p. 101044.

[16] Rajagambeeram, R., Malik, I., Vijayan, M., Gopal, N., Ranganadin, P. Evaluation of Serum Electrolytes and Their Relation to Glycemic Status in Patients with T2DM. Int J Clin Biochem Res. 2020; Vol. 7 (1): p. 130-133.

[17] Karuppan, A., Sahay, MI., Ravindranathan, R., Haripriya, P., Sriram, DK., George, M. Electrolyte Disturbances Among Diabetic Patients Admitted in A Multi-Specialty Hospital in Southern India. J Clin Diagn Res. 2019; Vol. 13 (2): p. OC12-OC15.

[18] Kanduker, S., Ahmed, R., Khondker, F., Aharama, A., Afrose, N., Chowdhury, MAA. Electrolyte Disturbance in Patients with Diabetes Mellitus. Bangladesh J Med Biochem 2017; Vol. 10 (1): p. 27-35.

[19] Woyesa, SB., Gebisa, WC. Anshebo, DL. Assessment of Selected Serum Electrolyte and Associated Risk Factors in Diabetic Patients. Diabetes Metab Syndr Obes. 2019; Vol. 12: p. 2811-2817.

[20] Kataoka, H., Yoshida, Y. Enhancement of The Serum Chloride Concentration by Administration of Sodium- Glucose Cotransporter-2 Inhibitor and Its Mechanisms and Clinical Signifcance in Type 2 Diabetic Patients: A Pilot Study. Diabetol Metab Syndr. 2020; Vol. 12 (5): p. 1-7.

[21] Palmer, BF., Clegg, DJ. Electrolyte and Acid-Base Disturbances in Patients with Diabetes Mellitus. N Engl J Med. 2015; Vol. 37 (3): p. 548-559. 